\title{
INTRINSIC SAFETY AND POSITIVE SECURITY WITH AN EMBEDDED SOFTWARE SOLUTION FOR EQUIPMENT USED IN SPACES WITH AN EXPLOSIVE POTENTIAL ATMOSPHERE
}

\author{
Gabriel Ioan Ilcea ${ }^{1}$, Pop Emil ${ }^{2}$, Ionut-Alin Popa ${ }^{3}$
}

\begin{abstract}
In this paper intrinsic safety and positive security distance control with an embedded software solution used in spaces with an explosive potential atmosphere is approached, in comparison to those which use just hardware technology. In sectors with an explosive potential atmosphere, a combined mixture of flammable materials, oxygen, and the presence of an ignition source (mostly caused by electric devices) can cause an explosion. For this reason, in areas with a risk of explosion the electrical equipment is made using an anti- explosive safety for the inputs and outputs called intrinsic safety. Currently this protection is made using a hardware solution which has a lot of drawbacks. To diminish or to get rid of these drawbacks and to ensure the safety of the workers and reduce material loses, the author developed and presented a method of protection for the electrical equipment using intrinsic safety and positive security combined with a software solution to create a safety area consisting in a device with a single button, controlled by a VLSI chip or PLC device and inserted into a metal housing.
\end{abstract}

UDC Classification: 004.35; DOI: http://dx.doi.org/10.12955/cbup.v6.1297

Keywords: explosive potential, explosion risk, intrinsic safety, positive security, software embedded, distance control.

\section{Introduction}

There are human activities that take place in dangerous conditions, and there are risks of work accidents. The most difficult situations are encountered in the following sectors: mining extraction, oil and chemistry, the marine sector, the army, food industry, etc. In these sectors explosive mixtures may accumulate and under the action of a flame or a simple spark can explode causing material damage or human victimization (Gaman, 2014). In this paper we will refer to the most unfavourable situations caused by electrical equipment that can initiate explosions if they are unprotected and work in atmospheres with accumulation of inflammable mixtures. We will present and develop the method of protection known as intrinsic safety and positive security that combined with a software solution is the goal of this paper.

The following concepts are defined in this paper: safety area, potentially explosive atmospheres area, explosion risk area, hazardous area, intrinsic safety, positive security and anti-explosive/ flameproof. Currently in hazardous areas the electrical equipment is made anti-explosive/ flameproof with intrinsically safe and positive security for all inputs and outputs. To ensure a speed of reaction in dangerous conditions, today the intrinsic safety in regard to positive security devices are ensured through the hardware (Dhillon, 2008). The hardware solutions haves many drawbacks. For this reason, in this paper a software-oriented solution was developed to replace the old one. The proposed solution consists of a device with a single-button, controlled by embedded software and, which is easy-to-implement using both a VLSI chip or a PLC device. The paper presents the algorithm and the main code part made in the VHDL language and tested on the simulator with the expected results validating the solution. At the end of the paper, the equipment with distance-control, intrinsically safety and positive security, controlled by the software was used in an application.

In conclusion, intrinsic safety and positive security with embedded software is a modern solution with many advantages which is justified to be considered for the design of such equipment.

\section{Software embedded principle for intrinsic safety and positive security}

At first, we will be establishing the conditions in which a location is considered to have explosive potential and risk of explosions. There are three conditions as follows: If there exist enough oxygen in the location's atmosphere for explosions; If there exist an easy inflammable materials in the location atmosphere; If there exist a source of energy which can initiate in some consequences the explosions. (Csaszar et al, 2011). All three conditions presented define the so called Explosion's triangle, Figure 1.

\footnotetext{
${ }^{1}$ Department of Control Engineering, Computers, Electrical Engineering and Power Engineering, University of Petroșani, Romania, gabrielilcea@upet.ro

${ }^{2}$ Department of Control Engineering, Computers, Electrical Engineering and Power Engineering, University of Petroșani, Romania, EmilPop@upet.ro

${ }^{3}$ Department of Control Engineering, Computers, Electrical Engineering and Power Engineering, University of Petroșani, Romania, ionutalin_popa@yahoo.ro
} 
The presence of first condition defines the normal working location. The presence of first two conditions in a location defines a potential explosive atmosphere. The presence of all three conditions defines a hazardous area with a risk of explosion. Some substances as gazes (methane, acetylene butane, propane, carbon monoxide), inflammable liquids (oils, chemical solvents, petrol, fuels), inflammable dustiest and plastic materials (cereals, wood, coal, sugar), change a normal location in one with potential explosive atmosphere (Leroux, 2005).

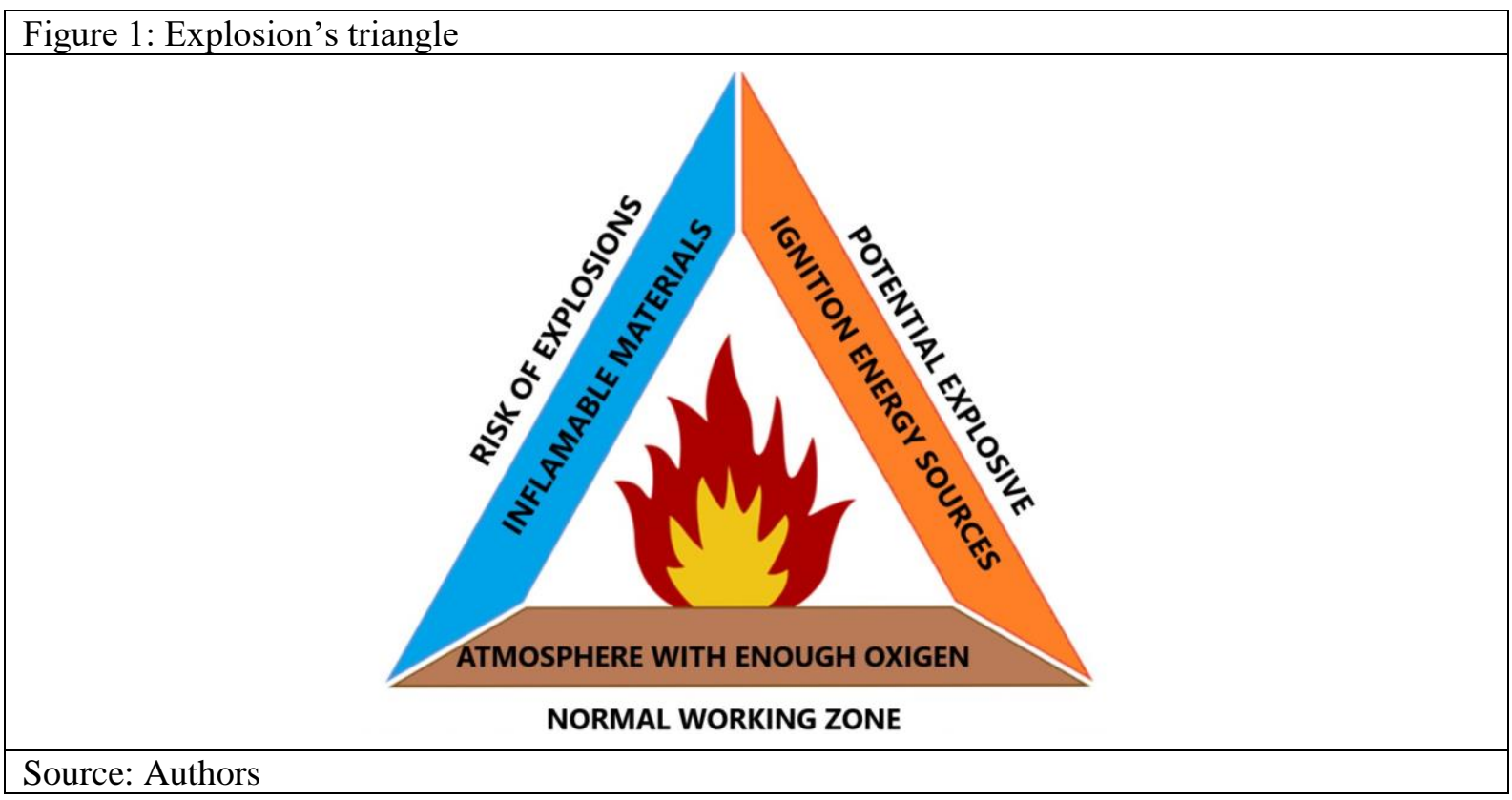

The presence of equipment with energy sources (electrical and/or mechanical spark, hot gases and flames, static electricity, electromagnetic waves, and chemicals reactions) change a potential explosive atmosphere in one with a risk of explosions. (Cioclea et al, 2008). In the following the concepts used in spaces with explosive potential atmosphere will be defined:

- An area where there exists a mixture of inflammable gases and oxygen in an adequate percentage for an explosion to occur is called an Explosive potential atmosphere;

- Any action which produces caloric energy capable to initiate an explosion is called an initiation source.

- An aria where there exists a mixture of inflammable gases and oxygen in an adequate percentage for an explosion to occur together with an initiation source is called a risk of explosion atmosphere;

- A zone where there does not exist any explosive potential atmosphere or cannot be transmitted from the initiation source for the explosion to the outside zone, is a safety area;

- An area with a risk of explosion atmosphere or an explosive potential atmosphere is called a hazardous area. A hazardous area is a zone presenting a risk of explosions. The electric equipment working in a hazardous area must be anti-explosive/anti-deflagrate or much better with intrinsic safety (Darie et al, 2010);

- A level of safety of electric equipment enclosed in a special carcass which never transmit from inside to outside an initiation source, have anti explosive /anti deflagrate safety;

- Electric equipment which in normal function or in defect state, cannot become an initiation source of an explosion have intrinsic safety (Bolton, 1998);

- An equipment that works in a safe area and is not capable of transmitting through its outputs a current that can ignite a flammable mixture, and haves positive security for its inputs, is called a security barrier.

To assure safety against explosions, technological and management resources are used. Therefore, electrical equipment are manufactured with dedicated anti-explosive measures and for underground mining with anti-deflagrate housing. An important category of electric equipment which is protected for any explosion risk are those with intrinsic safety which are manufactured with holistic safety and positive security too (Bottrill et al, 2005). 
In Figure 2 there are considered two working locations, first (on right) is a normal working location because it is a safety area or without risk of explosions. Here normal equipment are used which in some consequences can become sources of ignitions, but because the area is without potential explosive atmosphere, it remains the safe area. In the left of Figure 2 is a hazardous area where the equipment must be special manufactured with anti-explosive/ anti-deflagrate carcass, intrinsic safety and positive security for all its inputs/outputs. If we have to do the control from safety area to hazardous area the signals must be connected through safety barriers or use the equipment with intrinsic security.

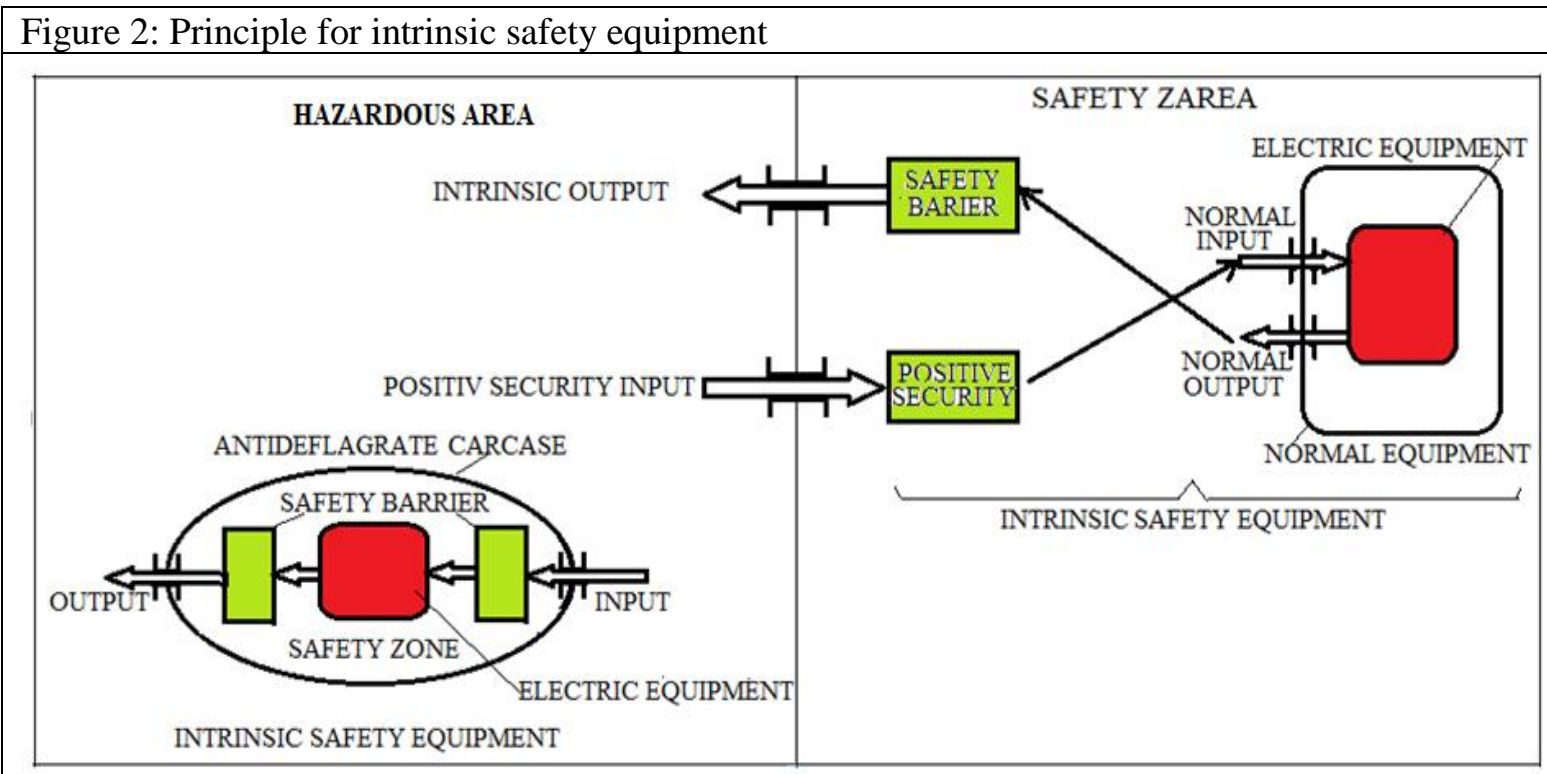

Source: Authors

To use the electric equipment in a hazardous area, a mini safety zone is created using a special enclosed carcass and the inputs and outputs are connected by safety barriers, like explained before. From the inputs and outputs of this equipment will flow such a low energy level which cannot be an initiation source (Ilcea 2014). The next important problem is distance control of inputs and positive security. As an example, in underground coal mining, a hardware device is used to distance control input. - This device- , is controlled by a passive button connected through a long cable and has intrinsic safety, positive security, and minimum conductors in the cable. In order to have a rapid speed of reaction, and working with all input in parallel, the device is is now made with a hardware structure. This solution has a lot of disadvantages like: low reliability and flexibility as well as high cost. To reduce the disadvantages presented above a solution based on oriented software will be developed (PNCDI-CEEx Project, 2005). This is the main motivation of this paper.

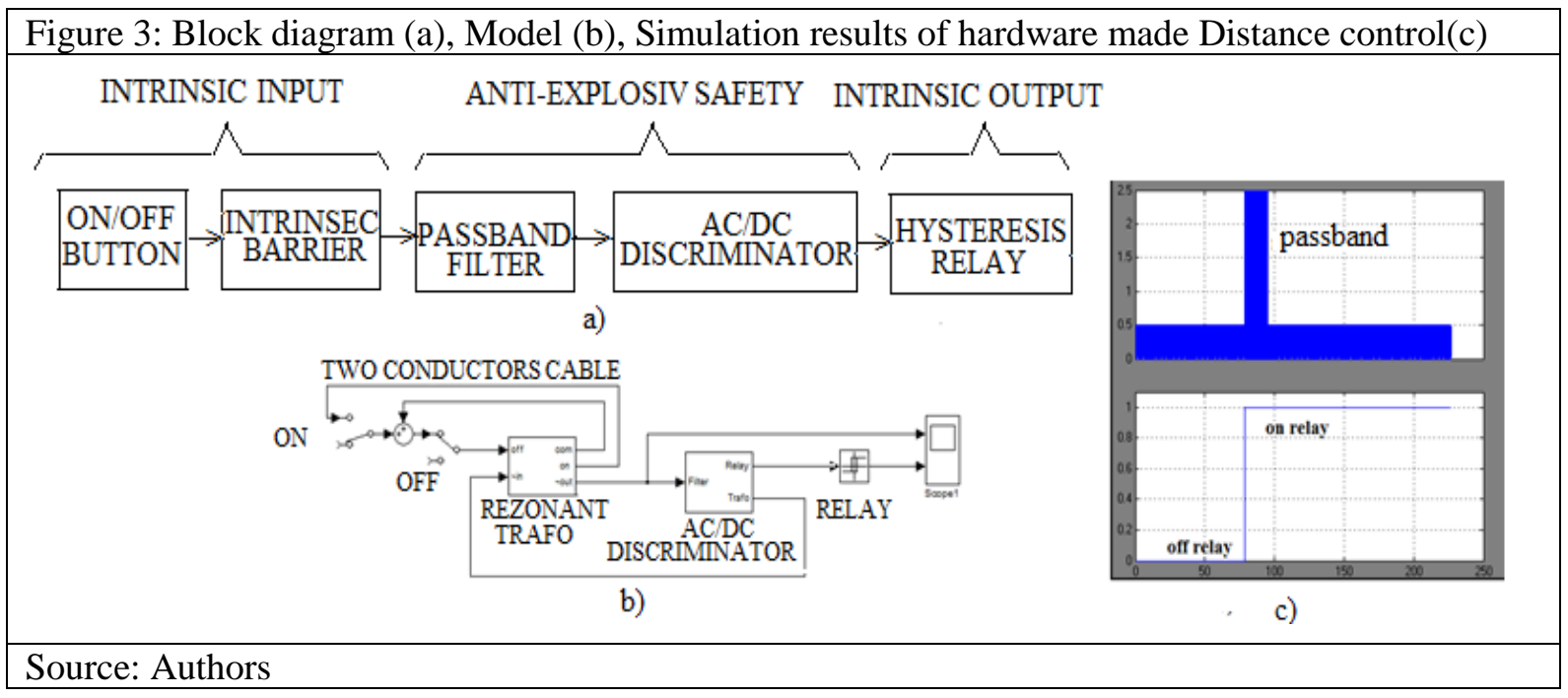




\section{Algorithm and software for intrinsic safety and positive security distance control}

The principle of distance control now used is based on hardware with some disadvantages compared with others which use microprocessors or PLC controlled through embedded software. The requirement for the equipment used in such conditions are anti-explosive/anti-deflagrate housings. An embedded software with minimum hardware will be a better solution which can cover all the requirements for this kind of equipment (Pop, 1993). In Figure 3 we present a block diagram, the model and the simulation results for the existing hardware solution used now, to see if a new approach is possible.

Analysing the block diagram of Figure 3a and the Simulink model of Figure 3b shows that input is intrinsic with a passive on/off push button placed on the first part of device. The output of the device consists of two NO/NC contacts of a DC relay and have intrinsic safety too. The state of the device is memorized through a shunt resistance placed in the button together with hysteresis of DC relay as shown in Figure 3c. This structure of the device assures the following qualities:

- The input and output use security barriers made with diodes and resistors to be in intrinsic security. The distance connection cable between the on/off button and device use only 2 conductors;

- The device has positive security which means: if the connection cable is cut or shorted the device disconnects the output relay contacts. We can conclude about the principles of the device discussed above as "Intrinsic safety and positive security".

The simulation results open the possibility to design the device using a software solution for positive security. In this case it will generate through a cable a sample code for the start and stop and the security barriers will be improved by using the optocouplers for assuring the intrinsic safety (Poantă et al, 2009).

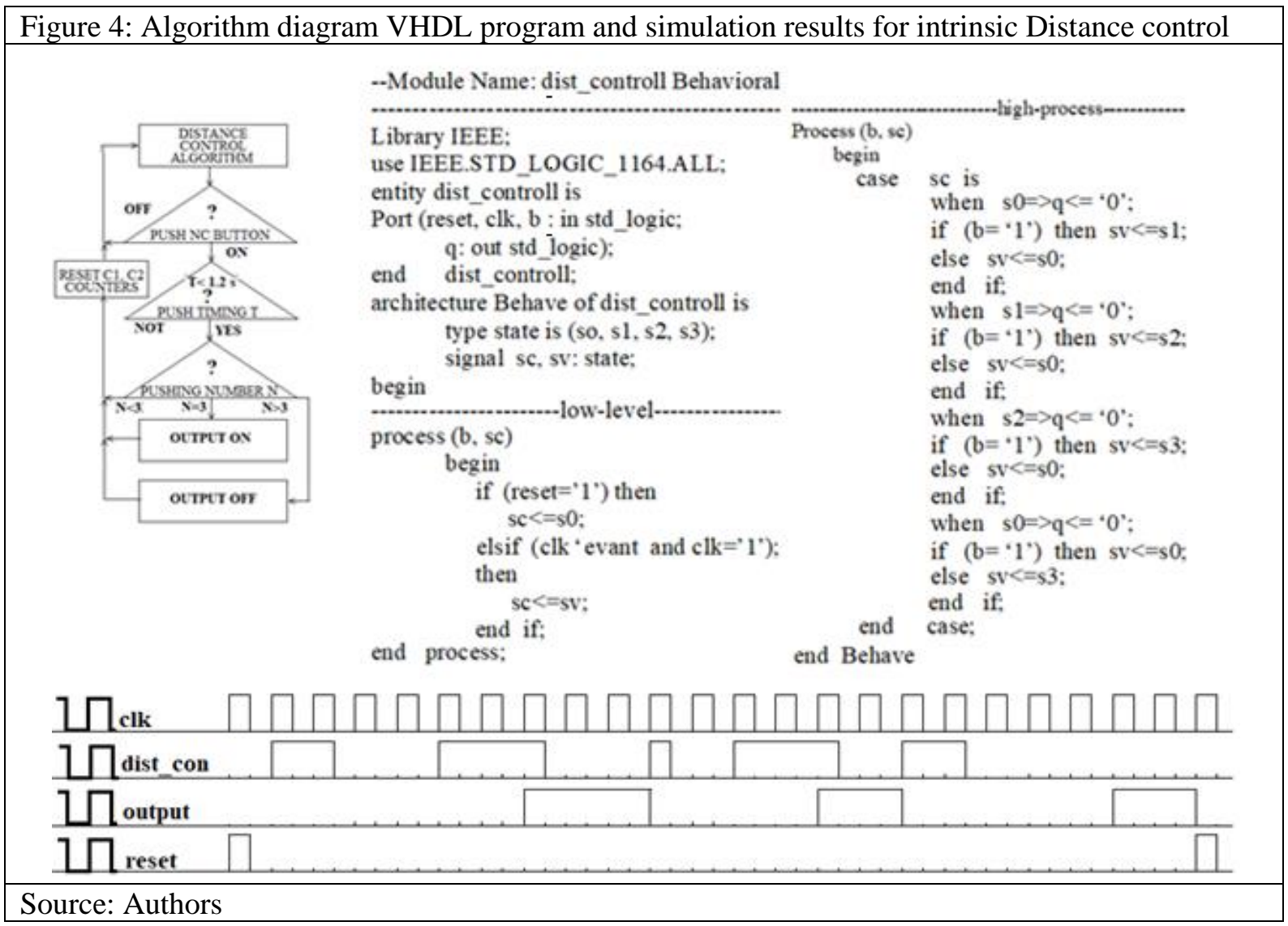

The two on/ off buttons will be replaced with one button in a NC position and the current control flows through it in a normal state. This single button has the same actions as the previous two, because it is controlled by software using a simple code. If the button is pushed three times, there is an ON state but if it is only pushed once or twice there will be an OFF state. In that mode the current control 
become the input and output signals code which is controlled from the distance by only one single button (Douglas, 2002). The device assures the before conditions and has additionally several advantages:

Reduced hardware through the use of a single button with two functions, software is integrated in equipment etc. (Pinto V. et al, 2007). In Figure 4 the algorithm logic diagram is presented as well as the-, VHDL program for a CPLD chip and simulation results using ISE-XILIX simulator for the distance control device as discussed above.

Software simulates the on-delay timing relay $\mathrm{T}$ with one retriggering input in an interval time of $(0.8 \ldots 1.4)$ seconds, and counters $\mathrm{C} 1, \mathrm{C} 2$. Input is sent by the NC push button on a rising signal. If the number of signals is only three the output $\mathrm{Q}$ is set $\mathrm{ON}$ and remains in this state, otherwise it becomes OFF. The OFF state also simulates the situation when the cable is cut or the case of a short-circuit. This principle can be used to design a standalone device with minimum hardware using a VLSI chip (Kenneth, 2014). A version of such a device with minimum hardware (ON/ OFF button) and having the software version described above integrated into a PLC can be seen in Figure 5.

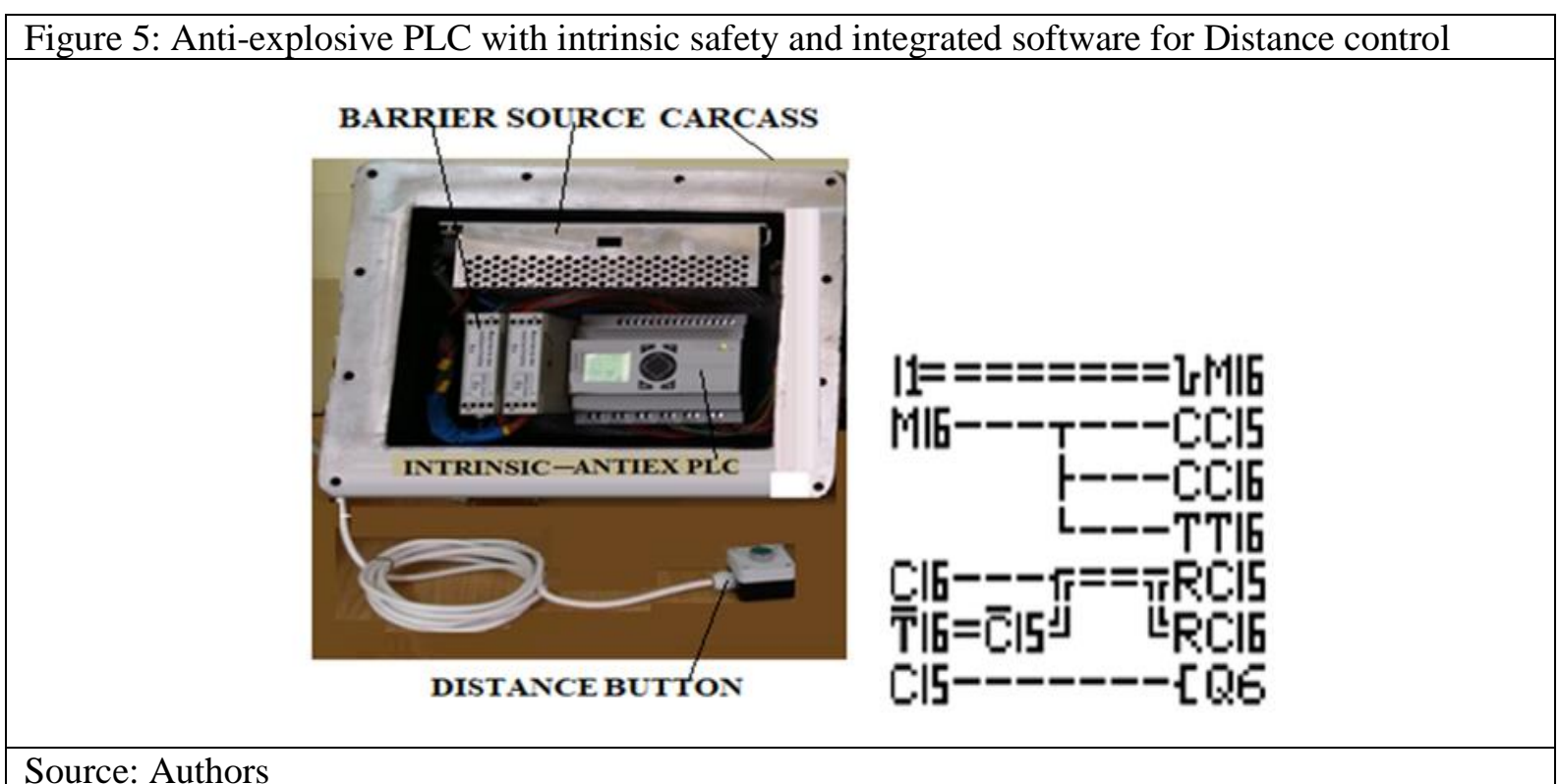

This solution is very comfortable because the programs with Ladder diagrams need a minimum number of instructions lines (Dunning, 2006). In Figure 5 an Anti-explosive PLC made with a Moeller device and a security barrier on its 12 inputs is presented. The 6 outputs are made with contacts set to be on passive energy. On an input is placed on a distance control button controlled by the software integrated into the PLC. The program was written in the Ladder diagram where I1 is the input, M16 is the software relay, CC15, CC16 are counters, TT16 is the timing relay and Q6 is the output. This equipment has intrinsic safety on its inputs/outputs and is enclosed in an anti-explosive carcass.

\section{Conclusion}

The following concepts are defined in this paper: safety area, potentially explosive atmospheres area, explosion risk area, hazardous area, intrinsic safety, positive security and anti-explosive/antideflagrant safety.

Currently in hazardous areas, electrical equipment must be made with anti-explosive/anti- deflagrant safety measures that have intrinsically safety and positive security for all their inputs and outputs. To ensure a fast speed of reaction in dangerous conditions, the intrinsic safety with positive security devices are made through hardware solutions today. The hardware solutions haves many drawbacks. A software-oriented solution to replace the old one was developed in this paper. This is a device with a single-button, controlled by embedded software and which is, - easy-to-implement using both a VLSI chip or a PLC device. The paper presents the algorithm and the main code part made in the VHDL language and which was tested on a simulator with the expected results validating the solution. At the end of the paper an anti-explosive/anti-deflagrate PLC with intrinsic safety inputs/outputs with software integrated for distance control is presented. 
This paper is dedicated to people who are working in dangerous sectors with potentially explosive atmospheres like mining, the army, the marine sector, oil and gas extraction, chemical industry etc. which can cause serious work accidents.

\section{References}

Bolton W. (1998). Control engineering, 2. London: Prentice Hall.

Bottrill G., Cheyne D. \& Vijayaraghavan G. (2005). Practical electrical equipment and installations in hazardous areas. Elsevier.

Cioclea, D., Toth, I., Lupu, C., Jurca, L. \& Gligor, C. (2008). Coal susceptibility to spontaneous combustion. Petrosani: INSEMEX Publishing House.

Csaszar T., Păsculescu D., Burian S., Darie M. \& Ionescu J. (2011). Method of assessment for energy limited supply sources, designed for use in potentially explosive atmospheres. International Symposium, Occupational Health and Safety (SESAM 2011), 5, Pages. 208-215.

Darie M., Burian S., Ionescu J., Csaszar T., Moldovan L. \& Andriş A. (2010). Modern prediction methods in the monitoring process of security parameters", WSEAS Transactions on Systems, 9 (7), Pages 713-723.

Dhillon B.S. (2008). Mining Equipment Reliability, Maintainability, and Safety. Springer Series in Reliability Engineering.

Douglas L. P. (2002). VHDL Programming by examples, 4. USA: MC Grow-HILL Corporation.

Dunning G., (2006). Introduction to programmable logic controller. Elsevier.

Gaman G. A., (2014), Symbiosis of environmental protection and occupational safety in toxic, explosive and flammable atmospheres: current knowledge and advances, Environmental Engineering and Management Journal, 13, Pages.1327-1328. Ilcea G., Dobra R., Păsculecu D. \& Buică G. (2014). Decision support system based on fiber optic technology applicable to mining industry. Recent Advances in Circuits, Systems, Signal Processing and Communications Proceedings of International Conference on Circuits, Systems, Signal and Telecommunications (CSST'14), Pages 148-151.

Kenneth L.S. (2014). VHDL for engineering. London:Pearson New International Edition

Leroux P. (2005). The operator's viewpoint of the ATEX directive 94/9/EC new regulations and rules for explosive atmospheres in Europe. Industry Applications Society 52nd Annual Conference on Petroleum and Chemical Industry, Pages $367-376$.

Pinto V., Silviano R. \& Martins J. F.. (2007). PLC controlled industrial processes on-line simulato", Industrial Electronics, 2007. ISIE 2007, Pages 2954-2957.

Poantă A., Dojcsar D. \& Sochirca B. (2009). System command of a pump instalation based on a programmable controller. Mine Review (Revista minelor) , 15, Pages 15-18.

Pop E. (1993). Automation in the mining industry. Bucharest: Didactic and Pedagogical Publishing House.

PNCDI-CEEx Project. (2005). Development of hardware and software structures in accordance with European requirements on the Safety Integrity Level-SIL - in the field of primary extractions and processing of oil and gas aimed for diminishing environmental impact. CEEx Research Program. 